\title{
LIMITATIONS IN THE DIAGNOSIS OF MOG ANTIBODY-ASSOCIATED DISEASE: A CASE REPORT
}

Paula Consoline Micheletto ${ }^{1}$,, Isabela Oliveira Vieira da Silva', Nabila Triântala Bellezzia de Salles ${ }^{1}$, Antonio Lucas Lima Rodrigues ${ }^{1}$, Maraísa Centeville', Roberto Marini', Simone Appenzeller ${ }^{1}$

1. Universidade Estadual de Campinas, Campinas (SP), Brazil.

*Corresponding author: pconsoline@gmail.com

\section{BACKGROUND}

Myelin oligodendrocyte protein (MOG) is a protein expressed only on the outermost lamellae of the myelin sheath and on the surface of oligodendrocytes, and, thus, is specific to the central nervous system (CNS). Anti-myelin oligodendrocyte protein is now considered to represent a biomarker of a distinct disease entity. Emerging data suggests anti-MOG seropositivity is directly pathogenic and has distinct neuroinflammatory and clinical features associated with unique histopathologic features. Only in 2015, the disease entity has been named as MOG antibody-associated disease (MOGAD). Since many rheumatic diseases present with central nervous system (CNS) manifestations, MOGAD is an important differential diagnosis.

\section{CASE REPORT}

A 14 years-old female patient, referred to our service at 6 years old due to epileptic seizures, negative ANA, drowsiness, daily fever, headaches, behavioral changes and abdominal pain. Differential diagnosis investigated during the follow-up years included systemic lupus erythematosus and Behçet's disease. Cerebrospinal fluid (CSF) unaltered, magnetic resonance imaging (MRI) with multifocal lesions and signs of demyelination, electroencephalogram with mild to moderate background activity, negative rheumatoid factor, negative antinuclear factor, increased complements, anti-Sm, anticardiolipin, deltaminolevulinic acid (ALAU), during remission, and porphobilinogen negative, negative screening for infectious disease, and normal ophthalmological evaluation. She evolved with relapsing disease course, presenting worsening of MRI, vomiting, changing in mental status, visual hallucinations and emotional lability. Central nervous system vasculitis was considered a possible hypothesis. She presented improvement with corticosteroid dose and 12 infusions. She improved clinically, but had subsequently flares. In 2018, she presented demyelination of both optic nerves, negative antiaquaporin- 4 and positive anti-MOG tests. Azathioprine was prescribed and the patient evolved with improvement of symptoms.

\section{CONCLUSION}

MOGAD is an important differential diagnosis of rheumatic diseases involving CNS. Further research is needed to better characterize this entity and optimal therapeutic approaches. 